\section{Delay-Dependent Anti-Windup Loops for Enlarging the Stability Region of Time Delay Systems With Saturating Inputs}

\section{S. Tarbouriech}

L.A.A.S.-C.N.R.S., 7 Avenue du Colonel Roche, 31077 Toulouse cedex 4, France

e-mail: tarbour@laas.fr

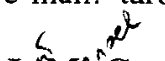

Jo M: Gomes da Silva, Jr.

UFRGS-Department of Electrical Engineering, Av. Osvaldo Aranha 103, 90035-190 Porto Alegre-RS, Brazil e-mail: jmgomes@eletro.ufrgs.br

\section{G. Garcia}

L.A.A.S-C.N.R.S., 7 Avenue du Colonel Roche,

31077 Toulouse cedex 4, France

e-mail: garcia@laas.fr

This paper addresses the problem of the determination of regions of stability for linear systems with delaved inputs and subject to inpul saturation through anti-uindup strategies. Differently of the most anti-uindup techniques, where the design of the anti-windup loop is introduced with the objective of minimizing the performance degradation. we are particularly interested in the synthesis of anti-windup gains in order to guarantee the stabilit of the closed-loop system for regions of admissible initial states as large as possible. With this aim, due to the presence of delay in the input uc propose delay dependent results. [DOI: 10.1115/1.1569953]

\section{Introduction}

Consider the linear continuous-time delay system:

$$
\left\{\begin{array}{l}
\dot{x}(t)=A x(t)+B u(t-\tau) \\
y(t)=C x(t)
\end{array}\right.
$$

with the initial conditions

$$
x\left(t_{0}+\theta\right)=\phi_{x}(\theta), \forall \theta \in[-\tau, 0], t_{0}, \phi_{x} \in \mathfrak{R}_{+} \times C_{\tau}^{\nu}
$$

where $x(t) \in \mathfrak{R}^{n}, u(t) \in \mathfrak{R}^{m}, y(t) \in \mathfrak{R}^{P}$ are the state, the input and the measured output vectors, respectively. Matrices $A, B$ and $C$ are real constant matrices of appropriate dimensions.

Considering system (1), we assume that an $n_{c}$-order dynamic output stabilizing compensator described by

$$
\begin{aligned}
& \dot{\eta}(t)=A_{c} \eta(t)+B_{c} y(t) \\
& \nu(t)=C_{c} \eta(t)+D_{c} y(t)
\end{aligned}
$$

where $\eta(t) \in \mathfrak{R}^{n_{c}}$ is the controller state, $y(t)$ is the controller input, and $\nu(t)$ is the controller output, has been determined. In fact, the control signal to be injected in the system is a saturated one, that is,

$$
u(t)=\operatorname{sat}(\nu(t))=\operatorname{sat}\left(C_{c} \eta(t)+D_{c} C x(t)\right)
$$

Contributed by the Dynamic Systems and Control Division of THE AMERICAN SOCIETY OF MECHANICAL ENGINEERS for publication in the ASME JOURNAL OF DYNAMIC SYSTEMS, MEASUREMENT, AND CONTROL. Manuscript received by the ASME Dynamic Systems and Control Division, May 2002; final revision, Jan. 2003. Associate Editor: N. Olgac. where each component of $\operatorname{sat}(\nu(t))$ are defined for $i=1, \ldots, m$ as: $\operatorname{sat}\left(\nu_{(i)}(t)\right)=\operatorname{sign}\left(\nu_{(i)}(t)\right) \min \left(\left|\nu_{(i)}(t)\right|, u_{0(i)}\right)$ where $u_{0(i)}>0$ denotes the control bound of the $i$ th input. In order to mitigate the undesirable effects of windup caused by input saturation, an antiwindup term $E_{c}(\operatorname{sat}(\nu(t))-\nu(t))$ can be added to the controller [1]. Thus, considering the dynamic controller and this anti-windup strategy, the closed-loop system reads:

$$
\begin{gathered}
\dot{x}(t)=A x(t)+B \operatorname{sat}(\nu(t-\tau)) \\
y(t)=C x(t) \\
\dot{\eta}(t)=A_{c} \eta(t)+B_{c} y(t)+E_{c}(\operatorname{sat}(\nu(t))-\nu(t)) \\
\nu(t)=C_{c} \eta(t)+D_{c} y(t)
\end{gathered}
$$

Let us now define the extended state vector

$$
\xi(t)=\left[\begin{array}{c}
x(t) \\
\eta(t)
\end{array}\right] \in \mathfrak{R}^{n+n_{c}}
$$

and the following matrices of appropriate dimensions

$$
\begin{gathered}
A=\left[\begin{array}{cc}
A & 0 \\
B_{c} C & A_{c}
\end{array}\right], \quad \mathrm{A}_{d}=\left[\begin{array}{cc}
B D_{c} C & B C_{c} \\
0 & 0
\end{array}\right], \quad \mathrm{B}=\left[\begin{array}{l}
B \\
0
\end{array}\right], \\
\mathrm{R}=\left[\begin{array}{c}
0 \\
I_{n_{c}}
\end{array}\right], \quad \mathrm{H}=\left[\begin{array}{ll}
D_{c} C & C_{c}
\end{array}\right]
\end{gathered}
$$

Hence, the closed-loop system reads:

$$
\dot{\xi}(t)=A \xi(t)+A_{d} \xi(t-\tau)-B(\mathbb{B} \xi(t-\tau))-\mathbb{R} E_{c} \psi(\mathbb{K} \xi(t))
$$

In Eq. (5), $\psi(\mathbb{K} \xi(t))=\nu(t)-\operatorname{sat}(\nu(t))=\mathbb{K} \xi(t)-\operatorname{sat}(\mathbb{K} \xi(t))$ is decentralized deadzone nonlinearity and satisfies the following sector condition [3]:

$$
\psi(\bar{h} \xi)^{\prime}[\psi(\mathfrak{K} \xi)-\Lambda \mathfrak{h} \xi] \leqslant 0, \quad \forall \xi \in S\left(\mathfrak{K}, u_{0}^{\lambda}\right)
$$

where $\Lambda$ is a diagonal matrix whose diagonal elements $\Lambda_{(i, i)}$ are simply denoted by $\lambda_{(i)}$, with $0 \leqslant \lambda_{(i)}<1$, and the polyhedral set $S\left(\bar{h} . u_{0}^{\lambda}\right)$ is defined by: $S\left(\mathbb{K}, u_{0}^{\lambda}\right)=\left\{\xi \in \mathfrak{R}^{n+n_{c} ;-u_{0(i)} / 1-\lambda_{(i)}}\right.$ $\left.\leqslant \AA_{(i)} \xi \leqslant u_{0(i)} / 1-\lambda_{(i)}, i=1, \ldots, m\right\}$.

The augmented system (5) admits an augmented initial condition

$$
\xi\left(t_{0}+\theta\right)=\phi_{\xi}(\theta)=\left[\begin{array}{l}
x\left(t_{0}+\theta\right)=\phi_{x}(\theta) \\
\eta\left(t_{0}+\theta\right)=\phi_{\eta}(\theta)
\end{array}\right], \quad \forall \theta \in[-\tau, 0]
$$

where $\phi_{\xi}(\theta)$ is supposed to satisfy $\left\|\phi_{\xi}\right\|_{c} \leqslant \nu, \nu>0$. Note that the initial condition of the dynamic output controller $\phi_{\eta}(\theta)$ is related to $\phi_{u}(\theta), \forall \theta \in[-\tau, 0]$ due to the relation $\phi_{u}(\theta)=C_{c} \phi_{\eta}(\theta)$ $+D_{c} C \phi_{x}(\theta)$.

It is important to note that the closed-loop matrix $\mathbf{A}$ will be asymptotically stable if and only if matrices $A$ and $A_{c}$ are asymptotically stable. Indeed, even if $A_{c}$ has been chosen asymptotically stable, $A$ may admit some unstable or not wished eigenvalues. In this case, we should study the stability of closed loop system (5) in a delay dependent context. System (5) is said globally asymptotically stable if for any initial condition satisfying $\left\|\phi_{\xi}\right\|_{c} \leqslant \nu$ with any finite $\nu>0$, the trajectories of system (5) converge asymptotically to the origin. Similar to the case of delay-free $(\tau=0)$, the determination of a global stabilizing controller is possible only when some stability hypothesis are verified by the open-loop system $(u(t)=0)[6]$. When this hypothesis is not verified, it is only possible to achieve local stabilization. The basin of attraction of system (5) corresponds to all initial conditions $\phi_{\xi}(\theta) \in \mathcal{C}_{\tau}^{v}$ such that the corresponding trajectories converge asymptotically to the origin. Since the determination of the exact basin of attraction is practically impossible, a problem of interest is to ensure the asymptotic stability for a set

$$
\Phi_{0}=\left\{\phi_{\xi} \in \mathcal{C}_{\tau}^{\nu} ;\left\|\phi_{\xi}\right\|_{c}^{2} \leqslant \delta\right\}
$$


of admissible initial conditions $\phi_{\xi}(\theta)$ [7]. Of course, the set $\Phi_{0}$ is included in the basin of attraction. Throughout the paper we refer a set $\Phi_{0}$ as a region of stability for system (5).

The problem we aim to solve throughout this paper is summarized as follows.

Problem 1: Determine the anti-windup gain matrix $E_{c}$ and $a$ scalar $\delta$, as large as possible, such that the asymptotic stability of closed-loop system (5) is ensured for all initial conditions $\phi_{\xi}(\theta)$ $\in \Phi_{0}, \forall \theta \in[-\tau, 0]$.

Since $\Phi_{0}$ can be viewed as an estimate of the basin of attraction of the system (5), the implicit idea behind Problem 1 is to enlarge this basin over the choice of the anti-windup gain matrix $E_{c}$. Throughout the paper, Problem 1 is addressed in the delay dependent context [5]. The measures and the criteria allowing to optimize the size of the region of stability of the closed-loop system, the tradeoff between this one and the delay magnitude will be discussed.

\section{Main Results}

In this section, we present a theoretical result, based on the verification of a set of matrix inequalities, that allows to compute an anti-windup gain $E_{c}$ and an associated region of stability for the closed-loop system (5).

Proposition 1: If there exist symmetric positive definite matrices of appropriate dimensions $W, X, R, H, U, D_{1}, D_{2}, D_{3}$, and $D_{4}$; diagonal positive definite matrices $\Lambda, G_{1}$, and $G_{2}$; a matrix $Z$; and a positive scalar $\gamma$ satisfying:

$$
\begin{aligned}
& Q=\left[\begin{array}{cccccc}
Q_{1} & Q_{2} & Q_{3} & Q_{4} & Q_{5} & Q_{6} \\
\star & Q_{7} & 0 & 0 & 0 & 0 \\
\star & \star & Q_{8} & 0 & 0 & 0 \\
\star & \star & \star & Q_{9} & 0 & 0 \\
\star & \star & \star & \star & Q_{10} & 0 \\
\star & \star & \star & \star & \star & Q_{11}
\end{array}\right]<0 \\
& {\left[\begin{array}{cc}
W & \left(1-\lambda_{(i)}\right) W \mathrm{~K}_{(i)}^{\prime} \\
\left(1-\lambda_{(i)}\right) \mathbb{K}_{(i)} W & \gamma u_{0(i)}^{2}
\end{array}\right] \geqslant 0, i=1, \ldots . m} \\
& 0 \leqslant \lambda_{(i)}<1, i=1, \ldots, m \\
& \text { where } Q_{1}=\left[\begin{array}{ccc}
\Gamma & \star & \star \\
\Lambda \mathrm{K} W-Z^{\prime} \dot{\mathrm{B}}^{\prime} & -2 G_{1} & \star \\
\Lambda \mathrm{K} W-G_{2} \mathrm{~B}^{\prime} & 0 & -2 G_{2}
\end{array}\right]<0 \text {. } \\
& \Gamma=W\left(A+A_{d}\right)^{\prime}+\left(A+A_{d}\right) W+\tau A_{d}(X+R+H+U) A_{d}^{\prime} . \\
& Q^{2}=\left[\begin{array}{cc}
\tau W A^{\prime} & 0 \\
0 & 0 \\
0 & 0
\end{array}\right], \quad Q_{3}=\left[\begin{array}{cc}
\tau A_{d}^{\prime} & 0 \\
0 & 0 \\
0 & 0
\end{array}\right], \\
& Q_{4}=\left[\begin{array}{cc}
0 & 0 \\
\tau G_{1} \mathrm{~B}^{\prime} & 0 \\
0 & 0
\end{array}\right], \quad Q_{5}=\left[\begin{array}{cc}
0 & 0 \\
\tau Z^{\prime} \mathbb{R}^{\prime} & 0 \\
0 & 0
\end{array}\right] \text {, } \\
& Q_{6}=\left[\begin{array}{c}
0 \\
0 \\
\tau \Lambda \mathrm{K}\left(D_{1}+D_{2}+D_{3}+D_{4}\right)
\end{array}\right] \text {, } \\
& \begin{array}{cc}
Q_{7}=\left[\begin{array}{cc}
-\tau X & \tau X \\
\star & -\tau\left(X+D_{1}\right)
\end{array}\right], \quad Q_{8}=\left[\begin{array}{cc}
-\tau R & \tau R \\
\star & -\tau\left(R+D_{2}\right)
\end{array}\right], \\
Q_{9}=\left[\begin{array}{cc}
-\tau H & \tau H \\
\star & -\tau\left(H+D_{3}\right)
\end{array}\right],
\end{array}
\end{aligned}
$$

Table $1 \delta$ for different values of $\tau$

\begin{tabular}{cccccc}
\hline \hline$\tau$ & 0.05 & 0.1 & 0.2 & 0.3 & 0.4 \\
$\delta$ & 3.7330 & 1.0297 & 0.2943 & 0.1429 & 0.0837 \\
$E_{c}$ & 0.1840 & 0.3027 & 0.7100 & 0.9718 & 0.4922 \\
$\Lambda$ & 0.8050 & 0.6560 & 0.4175 & 0.2397 & 0.09 \\
$\delta_{\text {lin }}$ & 0.1954 & 0.1912 & 0.1658 & 0.1233 & 0.0830 \\
$\delta_{E_{c}=0}$ & 2.9110 & 1.0274 & 0.2802 & 0.1361 & 0.0829 \\
\hline \hline
\end{tabular}

$Q_{10}=\left[\begin{array}{cc}-\tau U & \tau U \\ \star & -\tau\left(U+D_{4}\right)\end{array}\right], \quad Q_{11}=-\tau\left(D_{1}+D_{2}+D_{3}+D_{4}\right)$, then the gain matrix $E_{c}=Z G_{1}^{-1}$ and the set $\Phi_{0}=\left\{\phi \in \mathcal{C}_{2 \tau}^{\nu} ;\|\phi\|_{c}^{2}\right.$ $\leqslant \delta\}$ with $\delta=\gamma^{-1} / \delta_{N}$ and $\delta_{N}=\lambda_{\max }\left(W^{-1}\right)+3 \tau^{2} / 2\left[\lambda_{\max }\left(A_{d}^{\prime}\left(R^{-1}\right.\right.\right.$ $\left.\left.\left.+D_{2}^{-1}\right) A_{d}\right)+\lambda_{\max }\left(\mathrm{B}^{\prime}\left(H^{-1}+D_{3}^{-1}\right) \mathrm{B}\right)\|\Lambda \mathrm{K}\|^{2}\right]+\tau^{2} / 2\left[\lambda_{\max }\left(\mathrm{A}^{\prime}\left(X^{-1}\right.\right.\right.$ $\left.\left.\left.+D_{1}^{-1}\right) \mathrm{A}\right)+\lambda_{\max }\left(E_{c}^{\prime} \mathbb{R}^{\prime}\left(U^{-1}+D_{4}^{-1}\right) R E_{c}\right)\|\Lambda \mathrm{K}\|^{2}\right]$ are solutions to Problem 1 .

Proof: It is omitted for reasons of place but can be found in [2]. It uses Lyapunov-Krasovskii functional $V\left(\xi_{l}\right)=\xi(t)^{\prime} P \xi(t)+S\left(\xi_{l}\right)$. where $P=P^{\prime}>0$ and $S\left(\xi_{t}\right)$ is a positive definite quadratic form, and the fact that to ensure the stability of the closed-loop system (5), it suffices to ensure the stability for the following one [4]:

$$
\begin{aligned}
\dot{\xi}(t)= & \left(A+A_{d}\right) \xi(t)-B \psi(t-\tau)-R E_{c} \psi(t) \\
& -\int_{-\tau}^{0}\left[\lambda_{d} A \xi(t+\beta)-A_{d} B \psi(t-\tau+\beta)\right] d \beta \\
& -\int_{-\tau}^{0} A_{d}\left[-R E_{c} \psi(t+\beta)+A_{d} \xi(t+\beta-\tau)\right] d \beta
\end{aligned}
$$

with the initial data

$$
\xi\left(t_{0}+\theta\right)=\phi_{\xi}(\theta), \quad \forall \theta \in[-2 \tau, 0], \quad t_{0}, \quad \phi \in \mathfrak{R}_{-} \times \mathcal{C}_{2 \tau}^{\nu}
$$

\section{Computational Issues}

Proposition 1 provides constructive conditions for determining an anti-windup gain $E_{c}$ and a domain of initial states for which the closed-loop asymptotic stability is guaranteed. However. some bilinearities appear in the relations of Proposition 1 due to the product between $\Lambda$ and $W$. To overcome these difficulties, iterative schemes can be used. Furthermore. the implicit objective consists in obtaining a set $\Phi_{0}$ as large as possible. Thus, we can consider the following optimization criterion:

$\min \left\{\beta_{0} \gamma+\beta_{1} \operatorname{trace}\left(W^{-1}\right)+\beta_{2} \operatorname{trace}\left(H^{-1}+D_{3}^{-1}\right)+\beta_{3} \operatorname{trace}\left(\mathcal{A}_{d}^{\prime}\left(R^{-1}\right.\right.\right.$ $\left.\left.\rightarrow D_{2}^{-1}\right) A_{d}\right)+\beta_{4} \operatorname{trace}\left(A^{\prime}\left(X^{-1}+D_{1}^{-2}\right) A\right)+\beta_{5} \operatorname{trace}\left(U^{-1}\right.$ $\left.\left.+D_{+}^{-1}\right)\right\}$

where $\beta_{i}, i=0, \ldots, 5$ are weighting parameters. An algorithm based on some relaxations schemes and using the previous optimization criterion can then be proposed for providing a solution to Problem 1 (see more details in [2]).

\section{Illustrative Example and Concluding Remarks}

Consider the simple example borrowed from [3] in which the anti-windup problem is treated for $\tau=0$. System (1) is described by the following data:

$$
A=0.1 ; B=1 ; C=1 ; u_{0}=1
$$

and the dynamic controller is defined by:

$$
A_{c}=0 ; B_{c}=-0.2 ; C_{c}=1 ; D_{c}=-2
$$

The open-loop system is unstable since $A=0.1>0$. Thus matrix $A$ defined in Section 2 is also unstable, whereas matrix $\mathrm{A}+\mathrm{A}_{d}$ is asymptotically stable: $\operatorname{eig}\left(\mathrm{A}+\mathrm{A}_{d}\right)=-0.4 \pm j 0.2$. 
Table 1 shows the values of $\delta$ resulting from the application of the algorithm presented in [2] for different values of $\tau$. It indicates that we obtain greater $\delta$ for small $\tau$. Note that the values of $\delta$, denoted $\delta_{\text {lin }}$, obtained for each value of $\tau$ in the saturation avoidance case $\left(\Lambda=0, E_{c}=0, \psi=0\right)$ are always smaller than those obtained in the saturation allowance case. We can also notice that, in the case $E_{c}=0$, the values of $\delta$, denoted $\delta_{E_{c}}=0$, obtained for each value of $\tau$, are lower than in the case $E_{c} \neq 0$.

Furthermore, for $\tau>0.5$, it appears that the stability of the closed-loop system is only obtained in the linear case, that is the saturation is not allowed $(\psi=0)$ and the anti-windup strategy is not used $\left(E_{c}=0\right)$. Furthermore by searching the maximal value of the delay for which the linear closed-loop system $\xi(t)=\mathbf{A} \xi(t)$ $+\mathrm{A}_{d} \xi(t-\tau)$ is asymptotically stable one obtains: $\tau_{\max }=9.9989$.

\section{References}

[1] Cao, Y.-Y., Lin, Z., and Ward, D. G., 2002, "An Antiwindup Approach to Enlarging Domain of Attraction for Linear Systems Subject to Actuator Saturation," IEEE Trans. Autom. Control, 47(1), pp. 140-145.

[2] Tarbouriech, S., Gomes da Silva Jr., J. M., and Garcia, G., 2002, "DelayDependent Anti-Windup Loops for Enlarging the Stability Region of Time Delay Systems With Saturating Inputs," LAAS Report No. 02228.

[3] Tarbouriech, S., Gomes da Silva Jr., J. M., and Reginatto, R., 2002, "Analysis of Regions of Stability for Linear Systems With Saturating Inputs Through an Anti-Windup Scheme," Proc. of the IEE Conf. on Control Applications (CCA), Glasgow, Scotland. pp. 1106-1111.

[4] Hale, J. K., 1977, Theory of Functional Differential Equations. SpringerVerlag, New York.

[5] Niculescu, S.-I., 2001, Delay Effects on Stability. A Robust Control Approach. Springer-Verlag, Berlin, Germany.

[6] Saberi, A., Lin, Z., and Teel, A. R., 1996, "Control of Linear Systems With Saturating Actuators," IEEE Trans. Autom. Control, 41(3), pp. 368-378.

[7] Tarbouriech, S., and Gomes da Silva, Jr., J. M., 2000, "Synthesis of Controllers for Continuous-Time Delay Systems With Saturating Controls via LMIs," IEEE Trans. Autom. Control, 45(1), pp. 105-111.

\section{Preservation of the Full Relative Degree for a Class of Delay Systems Under Sampling}

\section{P. Pepe}

Dipartimento di Ingegneria Elettrica, Universitá degli Studi dell'Aquila, 67040 Poggio di Roio, L'Aquila, Italy e-mail: pepe@ing.univaq.it

This paper addresses the problem of the preservation of the full relative degree under sampling, for a particular class of nonlinear time delay systems, on the basis of the work by Barbot, Monaco, and Normand-Cyrot, ("A Sampled Normal Form for Feedback Linearization," Mathematics of Control, Signals and Systems, 1996. No. 9, pp. 162-188). The preservation of the relative degree, in order to build up the control law, has many advantages in the delayless case. The results of this paper are a first step in order to achieve the same advantages in the delayed case, too. [DOI: $10.1115 / 1.1570857]$

\section{Introduction}

The sampling problem for linear time-delay systems, has been addressed by many authors, and results are available in literature

Contributed by the Dynamic Systems and Control Division of THE AMERICAN SOCIETY OF MECHANICAL ENGINEERS for publication in the ASME JOURNAL OF DYNAMIC SYSTEMS, MEASUREMENT, AND CONTROL. Manuscript received by the ASME Dynamic Systems and Control Division. June 2002; final revision, January 2003. Associate Editor: N. Olgac. (see e.g., [1] and references therein), while, to my knowledge, the sampling problem for nonlinear time-delay systems has not been extensively addressed in literature. Papers are available in literature about sampling of nonlinear delayless systems (see for instance [2] and references therein). And results are available, too, for linearization and control of discrete time nonlinear systems (see, e.g., [3-7] and references therein).

In this paper, the sampling problem for a class of nonlinear time delay systems is addressed, on the basis of the methodologies shown in paper [2]. The discrete time equations of the approximated sampled nonlinear time delay system are found. Then, the main result is shown, that is, by using a suitable dummy output, the same given in [2], the full relative degree is preserved under sampling up to an approximation of order $n$ in the sampling period, with $n$ the length of the system variables $x(t)$.

This result can be successfully used to build up discrete time control laws for nonlinear time delay systems, as it is done for nonlinear delayless systems [2]. The great advantage of having a digital controller is evident, as far as the implementation is concerned. Take into account that a continuous time controller, which presents delays, is an infinite dimensional system, and its approximation generally is not a trivial task (see [8] and references therein).

An illustrative example is reported, showing the effectiveness of the digital control law built up by using the result of full relative degree preservation.

\section{Preliminaries}

Consider the following system

$$
\begin{gathered}
\dot{x}(t)=f(x(t))+g(x(t)) \\
\cdot\left(p_{1}(x(t), x(t-\Delta)) u(t)+p_{2}(x(t), x(t-\Delta))\right) \\
y(t)=h(x(t)), \quad t \geqslant 0
\end{gathered}
$$

with initial conditions $x(\tau)=x_{0}(\tau), \tau \in[-\Delta, 0]$, where $\Delta>0$ is an arbitrary, fixed delay, $x(t) \in \mathbb{R}^{n}, u(t) \in R$ and $y(t) \in R, f, g$ : $\mathrm{R}^{n} \notin \mathrm{R}^{n}, h: \mathrm{R}^{n} \notin: R, p_{1}, p_{2}: \mathrm{R}^{n} \times \mathrm{R}^{n} \notin \mathrm{R}$ are analytic functions, with continuous derivatives of any order.

From here on we suppose that the following hypothesis is satisfied by the system (2.1) (2.2):

$H_{p}$ ) the triple $(f, g, h)$ admits full uniform relative degree;

$$
\forall\left(\chi_{0}, \chi_{1}\right) \in \mathrm{R}^{n} \times \mathrm{R}^{n}, p_{1}\left(\chi_{0}, \chi_{1}\right) \neq 0 ;
$$

the function $\phi: \mathbb{R}^{n} \mapsto R^{n}$, defined by $\phi(x)$ $=\left[h(x) l_{f} h(x) \ldots l_{f}^{n-1} h(x)\right]^{T}, x \in \mathrm{R}^{n}$, is analytic and invertible, and the inverse $\phi^{-1}$ is analytic and $C^{\infty}$ too.

Remark 2.1: When the system (2.1) (2.2) satisfies the hypothesis $H_{p}$, then it admits full uniform delay relative degree (see [9]).

Let

$$
\begin{gathered}
z^{T}(t)=\left[\begin{array}{lll}
y(t) & y^{(1)}(t) \ldots y^{(n-1)}(t)
\end{array}\right]^{T}, t \geqslant 0, \\
z(\tau)=\phi\left(x_{0}(\tau)\right), \tau \in[-\Delta .0]
\end{gathered}
$$

Let us rewrite the system (2.1) (2.2) in normal form

$$
\begin{gathered}
\dot{z}(t)=A z(t)+B\left(l_{f}^{n} h\left(\phi^{-1}(z(t))\right)+l_{g} l_{f}^{n-1} h\left(\phi^{-1}(z(t))\right)\right. \\
\cdot\left(p_{1}\left(\phi^{-1}(z(t)), \phi^{-1}(z(t-\Delta))\right) u(t)\right. \\
\left.\left.+p_{2}\left(\phi^{-1}(z(t)), \phi^{-1}(z(t-\Delta))\right)\right)\right) \\
z(\tau)=\phi\left(x_{0}(\tau)\right), \tau \in[-\Delta, 0]
\end{gathered}
$$

where $A, B$ have the Brunowsky canonical form (see [10], pp. 153,231 ). For a suitable integer $m \geqslant 1$, let $T=\Delta / m$ be the sampling period. Let $z^{(i)}(t)$ denote the $i$-th time derivative of $z(t)$, $t \geqslant 0, i \geqslant 1$.

As in [2], we sample the system (2.4) by the Taylor formula 